\title{
Extracting Biological Pathway Models From NLP Event Representations
}

\author{
Michael Spranger * \\ Sony Computer Science \\ Laboratories Inc. \\ Tokyo, Japan \\ michael.spranger \\ @gmail.com
}

\author{
Sucheendra K. Palaniappan * \\ INRIA, \\ Campus de Beaulieu, \\ Rennes, France \\ sucheendra.palaniappan \\ dinria.fr
}

\author{
Samik Ghosh \\ The Systems Biology Institute, \\ Minato-ku, \\ Tokyo, Japan \\ ghoshesbi.jp
}

\begin{abstract}
This paper describes an an open-source software system for the automatic conversion of NLP event representations to system biology structured data interchange formats such as SBML and BioPAX. It is part of a larger effort to make results of the NLP community available for system biology pathway modelers.
\end{abstract}

\section{Introduction}

Biological pathways represent important insights into the flow of information within a cell by encoding the sequence of interactions among various biological players (such as genes, proteins etc.) in response to certain stimuli (or spontaneous at times) which leads to a change in the state of the cell. Studying and analyzing these pathways is crucial to understanding biological systems.

Traditionally, pathways are represented as maps which are constructed and curated by expert curators who manually read numerous biomedical documents, comprehend and assimilate the knowledge into maps. This process is aided by a variety of graphical tools such as CellDesigner (Funahashi et al., 2008).

Such manual pathway curation comes with a number of problems. Most importantly: 1) the amount of time and therefore cost for detailed pathway maps is high. 2) As new research findings are published these pathway need to be updated or augmented. Often, the speed at which molecular research is progressing, means it is hard to keep pathways in sync. 3) Many times the interpretation of details is left to the judgment of the curator, which leads to considerable variability of pathways.

Considering these limitations, there has been an increased emphasis on using Natural Language

These two authors contributed equally to this paper and the software system.
Processing (NLP) techniques for automated pathway curation. The BioNLP Shared Task - Pathway Curation (BioNLPST-PC) competition (Nédellec et al., 2013; Ohta et al., 2013) was focused on this specific problem. From the NLP perspective the extraction of biological knowledge is posed as an event detection problem with standard NLP event detection algorithms used to extract the biological information from text (Ananiadou et al., 2010).

Although there has been a lot of work on the problem of automatic pathway extraction from text, to our knowledge there has been little effort to make the extracted information available in standard pathway formats. The majority of pathway data is represented, stored and exchanged using standard formats such as SBML (Hucka et al., 2003) and BioPAX (Demir et al., 2010). Contrary to these formats existing NLP extraction systems often use a data format called the "standoff format", to represent their results. While the standoff format is often described as easily convertible into SBML and BioPAX, no actual software seems to exist to automate this conversion. This paper tries to fill this gap by describing a software system for the conversion of NLP event representations to the system biology structured data interchange formats SBML and BioPAX. We also provide open sourced software tools st2sbml and st2biopax to convert from stand-off to SBML/BioPAX format. The software tools and additional information about the contents of this paper can be found on our supplementary webpage $^{1}$.

\section{NLP Event Representations}

Existing NLP systems often use an event representation format comprised of a set of annotation rules and file formats to represent pathway events and entities (Kim et al., 2011). For the purpose of

\footnotetext{
${ }^{1}$ https://github.com/sbnlp/ standoff-conversion
} 


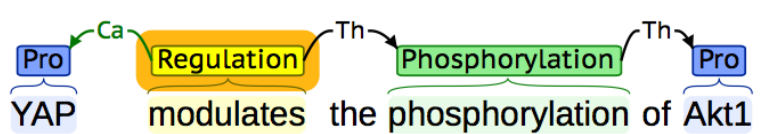

Figure 1: Graphical representation of the event representation of Example 1

this paper we base ourselves on the standoff representation (ST) proposed for the BioNLP Shared Task 2011, 2013 (Nédellec et al., 2013).

Annotations in ST link spans of texts through character offsets to entities (e.g. Proteins, Genes etc.) and events (Positive Regulation etc.). Events and entities are represented line by line with links between them.

The following is an example sentence and a possible event representations.

(1) YAP modulates the phosphorylation of Akt1.

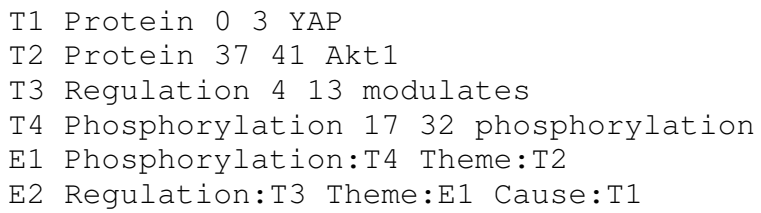

Each annotation starts with a unique annotationID. The annotations-IDs encodes the annotation type in the first letter ( $\mathrm{T}$ - text bound annotation, E - event annotation). This is followed by the annotation-type. For instance, the text bound annotation $\mathrm{T} 1$ is of type protein, whereas $\mathrm{T} 3$ is of type Regulation. Text bound annotations also encode the start and end position as well as the text they annotate. Text bound annotation T1 for instance ranges from character 0 to character 3 of the annotated text and the actual text is "YAP".

Event annotations build on top of text bound annotation. The annotations-ID for an event is followed by an event-type and the reference to the text bound annotation. For instance, E1 is a Phosphorylation event and the corresponding text is T4 "phosphorylation". Additionally, event annotations encode roles. T2 is the theme of E1, which in this case means that "Akt1" is undergoing a phosphorylation. Events can also be used as theme. For example the theme of E2 is E1, which means that the phosphorylation is regulated by "YAP". Different roles are possible depending on the type of the event.

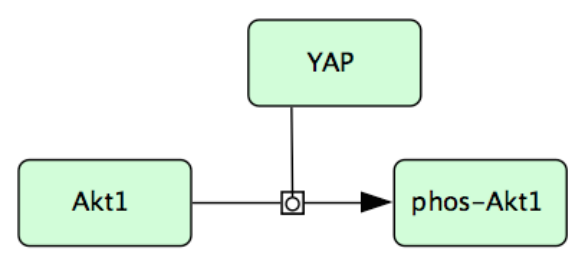

Figure 2: Example 1 converted into SBML (viewed with CellDesigner)

\section{From Event Representations to SBML}

Systems Biology Markup Language, or short SBML (Hucka et al., 2003), is a XML-based markup language to describe, store and communicate biological models. It is among the most widely used formats with numerous software support. SBML essentially encodes models using biological players called sbml: species ${ }^{2}$. sbml:species can participate in interactions, called sbml:reaction. Species participate in interaction as sbml:reactant, sbml:product and sbml:modifier. The basic idea being that some quantity of reactant is consumed to produce a product. Reactions are influenced by modifiers.

SBML supports mathematical representations of the underlying dynamics of the reactions and is essentially used to simulate models. Due to this, there is no SBML vocabulary to specify different types of reactions (such as transcription, phosphorylation etc.) or species (such as protein, DNA etc.). Alternatively, species and reactions can be annotated and uniquely specified using MIRIAM resources and annotations (Novere et al., 2005). We use controlled vocabulary from the Systems Biology Ontology (SBO) and the Gene Ontology (GO). This information is also useful to convert SBML files to other formats such as SBGN (Le Novere et al., 2009) using tools such as VANTED (Junker et al., 2006).

Figure 2 shows Example 1 converted into an SBML model using the mapping algorithm described in the following paragraphs.

\subsection{Mapping Algorithm}

The conversion of standoff formatted information to an SBML model consists of five steps.

\footnotetext{
${ }^{2}$ We will refer to SBML vocabulary using the prefix "sbml".
} 


\begin{tabular}{|c|c|c|}
\hline $\begin{array}{l}\text { Standoff } \\
\text { Entity }\end{array}$ & SBO term & SBO name \\
\hline Complex & SBO:0000253 & non-covalent complex \\
\hline $\begin{array}{l}\text { Gene_or } \\
\text { gene_product }\end{array}$ & SBO:0000245 & macromolecule \\
\hline Dna & SBO:0000251 & deoxyribonucleic acid \\
\hline DnaRegion & SBO:0000251 & deoxyribonucleic acid \\
\hline Drug & SBO:0000247 & simple chemical \\
\hline Ion & SBO:0000327 & non-macromolecular ion \\
\hline Protein & SBO:0000252 & polypeptide chain \\
\hline Rna & SBO:0000250 & ribonucleic acid \\
\hline RnaRegion & SBO:0000250 & ribonucleic acid \\
\hline Gene & SBO:0000354 & $\begin{array}{l}\text { informational molecule } \\
\text { segment }\end{array}$ \\
\hline Small & & \\
\hline Molecule & SBO:0000247 & simple chemical \\
\hline $\begin{array}{l}\text { Simple }_{-} \\
\text {molecule }\end{array}$ & SBO:0000247 & simple chemical \\
\hline
\end{tabular}

Table 1: Mapping of Annotation-type to SBO term.

Step 1: Initialize the Model Firstly, read the event annotation files and create a memory internal representation of triggers and events. We initialize an empty SBML model with a single sbml : compartment named "default".

Step 2: Create sbml:species For each entity in the standoff format, a sbml: species is added to the SBML model. This only applies to standoff entities that can be mapped to an SBO term. Then the following is done 1) map the annotationID of the trigger to the id in the sbml : species, 2) create a meta id by appending "metaid_0000" and annotation-ID; meta id facilitates that annotations to this species can uniquely refer to it 3) add the annotation-text as the name of the sbml: species, 4) map the annotation-type to an SBO term and add to the sbml : species (see Table 1)

For instance, the standoff line

T2 Protein 3741 Akt1

will be mapped to

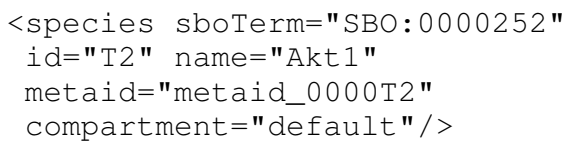

On the other hand, a line such as

T39 Entity 641648 nucleus

will not be used to create a species in the SBML model, because "Entity" cannot be mapped to an SBO term. Here, "nucleus" actually refers to a compartment which is not directly deducible from the entity definition in the standoff format. To deal with such cases, we need to take into account their role in Events something that is described in the next few paragraphs.

\begin{tabular}{|c|c|c|}
\hline Standoff Event & SBO/GO term & SBO/GO name \\
\hline Conversion & SBO:0000182 & conversion \\
\hline Acetylation & SBO:0000215 & acetylation \\
\hline Deacetylation & GO:0006476 & $\begin{array}{l}\text { Protein Deacetyla- } \\
\text { tion }\end{array}$ \\
\hline Methylation & SBO:0000214 & Methylation \\
\hline Demethylation & GO:0006482 & $\begin{array}{l}\text { Protein Demethyla- } \\
\text { tion }\end{array}$ \\
\hline Phosphorylation & SBO:0000216 & phosphorylation \\
\hline $\begin{array}{l}\text { Dephosphoryla- } \\
\text { tion }\end{array}$ & SBO:0000330 & Methylation \\
\hline Ubiquitination & SBO:0000224 & Ubiquitination \\
\hline Deubiquitination & GO:0016579 & $\begin{array}{l}\text { Protein Deubiquiti- } \\
\text { nation }\end{array}$ \\
\hline Degradation & SBO:0000179 & degradation \\
\hline Catabolism & GO:0009056 & Catabolic Process \\
\hline Catalysis & SBO:0000172 & Catalysis \\
\hline $\begin{array}{l}\text { Protein_- } \\
\text { catabolism }\end{array}$ & GO:0009056 & Catabolic Process \\
\hline Association & SBO:0000177 & $\begin{array}{l}\text { non-covalent bind- } \\
\text { ing }\end{array}$ \\
\hline Binding & SBO:0000177 & $\begin{array}{l}\text { non-covalent bind- } \\
\text { ing }\end{array}$ \\
\hline Disso & SBO:0000180 & dissociation \\
\hline Regulation & GO:0065007 & $\begin{array}{l}\text { biological regula- } \\
\text { tion }\end{array}$ \\
\hline $\begin{array}{l}\text { Positive } \\
\text { regulation }\end{array}$ & GO:0048518 & positive regulation \\
\hline Activation & SBO:0000412 & biological activity \\
\hline $\begin{array}{l}\text { Negative } \\
\text { regulation }\end{array}$ & GO:0048519 & negative regulation \\
\hline Inactivation & SBO:0000412 & biological activity \\
\hline $\begin{array}{l}\text { Gene_ } \\
\text { expression }\end{array}$ & GO:0010467 & Genetic Production \\
\hline Transcription & SBO:0000183 & Transcription \\
\hline Translation & SBO:0000184 & Translation \\
\hline Localization & GO:0051179 & Localization \\
\hline Transport & SBO:0000185 & Transport Reaction \\
\hline Pathway & SBO:0000375 & Process \\
\hline
\end{tabular}

Table 2: Mapping of annotation-type to SBO/GO term.

Step 3: Create sbml:reaction Most events are added to the SBML model as sbml: reaction. For instance, the text trigger and event annotation corresponding to E1 in Example 1 result in the following SBML description

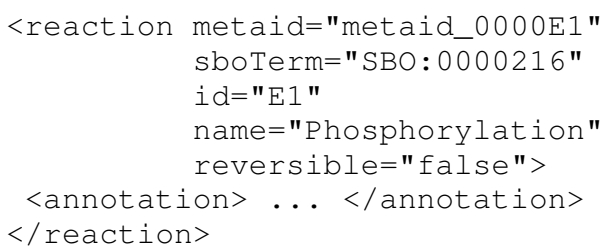

The $\mathrm{SBO} / \mathrm{GO}$ term is assigned according to the mapping depicted in Table 2. The reaction id is based on the event id (E10). The metaid of the form "metaid_0000 + id" is also added and the sbml:reaction name is the event-type. Lastly, all reactions are constructed as non reversible. 
In a second step sbml:reactant, sbml:product and sbml:modifier are added to SBML reactions based on the roles of events.

Theme is the entity that undergoes the effects of the event. It is mapped to the sbml: reactant of the SBML reaction. For this a reactant reference is created and the species corresponding to the entity is linked to that reference via the id of the species (annotation-id of the entity).

Product can be specified for Binding, Dissociation $^{3}$ and Conversion events. Product is mapped to sbml:product of the corresponding reaction. The entities appearing in the product role are used for creating a product reference with the same entity.

Cause is an entity/event causing the event. Cause is eventually mapped to entities which are then mapped to the reaction as sbml : modifier (via modifier reference).

Information in Site (which describes the site on the Theme entity that is modified in the event) is added to the "Notes" section of the SBML reaction as there seems to be no direct way to represent this information in SBML. Notes are human-readable annotations that can be added to SBML reactions.

\section{Step 4: Handle Localization and Transport} Events Localization and Transport events are handled differently from other events. They occur with additional roles besides Theme.

AtLoc describes the location/compartment at which the entity/species is located not an actual reaction. Hence, localization events with AtLoc roles do not end up as reactions in SBML. Instead, first we check if a sbml:compartment described by the AtLoc role exists, else a new sbml:compartment is created (see the nucleus example discussed earlier). Next, the compartment of the theme entity of the event is set to the corresponding sbml : compartment.

\footnotetext{
${ }^{3}$ In data used for evaluation we also encountered Dissociation events with Participant and Complex roles. They are mapped to sbml:product and sbml : reactant respectively.
}

FromLoc/ToLoc Transport and Localization events can also include FromLoc and TOLOC roles which describes the transport of the theme entity/species from some location/compartment to another. Consequently, we create a reaction where the Theme entity/species starts out in the compartment described by FromLoc (sbml:reactant) and ends up in the compartment described by the ToLOC (sbml:product) role. If the FromLoc/ToLoc sbml: compartment does not exist when creating the sbml:reaction, a new sbml:compartment is created corresponding to FromLoc/ToLoc.

Step 5: Handle Gene Expression Events We model Gene expression events (e.g. Transcription and Translation) as reactions in SBML. However, this class of reactions does not have the sbml : reactant role. For Transcription events (process in which a gene sequence is copied to produce RNA) if the type of Theme is RNA, it gets mapped to sbml:product. If the type of Theme is DNA, then it gets mapped to the sbml:modifier of the Transcription sbml: reaction.

Translation events are handled in a similar manner.

Step 6: Handle Regulation Events In principle regulation events such as Positive/Negative Regulation, Activation and Inactivation can be handled as described in Step 3 when the Theme and Cause are species. If Theme and Cause are species then they are added to a regulation reaction as reactant and modifier respectively.

However, the standoff format definition also allows regulation events where Theme and Cause are themselves events ${ }^{4}$. For example, the following standoff lines describe a Positive regulation of a Phosphorylation event.

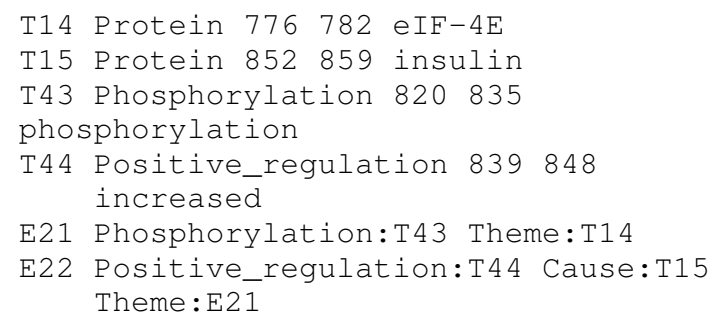

\footnotetext{
${ }^{4}$ In some of the data used to test our conversion we also encountered Catalysis events which had event themes. They are handled exactly as Positive Regulation events.
} 
If the Theme is an event, then we do not create a reaction but simply add the Cause entity as a modifier to the reaction corresponding to the Theme event of the regulation. For the example above this means that the Phosphorylation reaction E21 is positively regulated (modified) by insulin (T15).

In reality though things are a bit more complicated since the Theme event might itself not exist as a reaction. For instance, there could be an event description as follows:

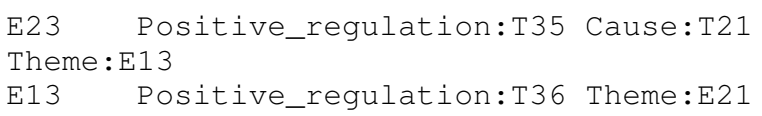

Here, the event E23 has Theme E13, which itself is a Positive regulation with Theme E21. However, E13 itself does not correspond to a reaction. In this case the algorithm recursively tracks down the Theme event across multiple event annotations until it finds an event that exists in the SBML model as a reaction (In this case E21 is identified as the Theme for E23).

In case the Cause is an event, the product of the Cause event is used as a modifier. If the reaction corresponding to the Cause event does not have a product yet, then a corresponding product species is first created and added to the model.

Step 7: Optional Cleanup and Annotation Operations As a last step optional cleanup/enhancement operations can be performed. They can be used to ensure consistency of the resulting SBML model.

Add UniProt information We use the annotation -text to retrieve information about species from UniProt. The UniProt ID is added as controlled vocabulary to the corresponding SBML species. Other information is added as XML annotation and XHTML notes. This includes information about alternate names, gene names, gene ids where available and appropriate.

Remove unused species Not all entities end up as products, reactants or modifiers of an SBML reaction. In many cases, the named entity recognizer might recognize some entity but no links to events is established. However, the entities might have been added to the model (see Step 1). Entities not partaking in any reaction can be removed automatically.
Complete reactions Our software supports automatic adding of products and reactants for reactions that were not explicitly annotated in that way. For instance, all phosphorylation events can extended with corresponding sbml:product species. The completion takes into account that certain reactions such as Gene expression reactions do not have reactants.

Here is an example of what we mean. For a Phosphorylation reaction, the first pass of the algorithm maps the Theme to sbml:reactant and no sbml:product is added. For example, E1 (in Example 1) would have Akt1 as a sbml:reactant. To complete this reaction a new sbml:species with name phoAkt1 is created representing the phosphorylated form of Akt. phoAkt1 is added as the sbml:product to the reaction E1 (See Figure 2).

\section{Remove reactions without reactants, products} In other defunct cases the standoff file might include events that cannot be translated into reactions with reactants and/or products. For example, we encountered in real data that a reaction might only have a modifier (Cause). Such reactions are automatically removed if requested by the user.

\subsection{Implementation}

We used python and the python version of libSBML to develop the conversion algorithm. libSBML was used for generating and accessing the SBML model content. We used a custom implementation of a Standoff parser which translates the line-wise description of standoff triggers and events in a1/a2 and ann files into a memory structure of triggers (id, type, text) and events (id, type, roles). These structures are the basis for generating and completing the SBML model. The conversion is fast. It scales linearly with the number of entities, events and roles.

\subsection{Discussion}

The conversion of standoff format files to SBML is quite straightforward with a few exceptions where events cannot be mapped directly to an SBML reaction as is the case with Localization events that have an AtLOC role. Moreover, not all entities end up as sbml : species. Cellular components 


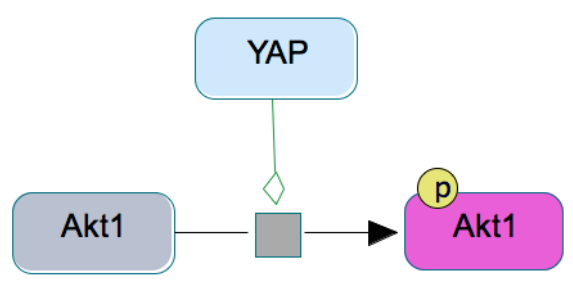

Figure 3: Example 1 converted into BioPAX (viewed with the ChiBE editor)

used in Localization and Transport events, for instance, end up as compartments. Another example are Regulation events that have events as Theme. In all of these cases, events in the standoff do not have a direct correspondent in the sbml model.

The algorithm is open to extension. For instance, in order to integrate a new event with Theme, Cause, Product, Site roles only a new SBO mapping needs to be defined.

SBML has graphical editing tool support through, for example, CellDesigner. Although CellDesigner uses SBML as its base format, there are a lot of tool specific custom XML annotations that convey a more fine grained view of sbml:species and sbml:reactions for visualization purposes. Our focus in this paper is the conversion to pure SBML format without toolbased customizations.

\section{From Event Representations to BioPAX}

Biological Pathway Exchange (BioPAX) is another widely used pathway data format based on $\mathrm{RDF} / \mathrm{OWL}$. It is used for storage, analysis, integration and exchange of pathway models (Demir et al., 2013) . BioPAX, unlike SBML is more fine grained in its explicit handling of different types of biological players (bp: Physicalentity ${ }^{5}$ ), and their interactions (bp: Interaction).

The BioPAX conversion algorithm is similar in structure to the SBML conversion. Figure 3 shows Example 1 converted into a BioPAX model using the mapping algorithm described in the following paragraphs.

Step 1: Initialize the model Read the event files, parse and create a memory internal representation of triggers and events. Create an empty model BioPAX model.

\footnotetext{
${ }^{5} \mathrm{We}$ will henceforth use the the prefix bp to refer to BioPAX vocabulary
}

Step 2: Create bp:PhysicalEntity Each entity in the standoff format is mapped to the corresponding bp:Physicalentity class in BioPAX. bp:Physicalentity is a superclass of molecules such as proteins, DNA, RNA, Small molecules, Complex etc. Depending on the granularity of the description of the entity, the element is initialized as a sub class of bp:Physicalentity. The mapping is described in Table 3. The created $\mathrm{bp}$ :Physicalentity is assigned a unique-id which is the same as the annotation-ID of the entity. The name of the bp:Physicalentity is assigned the annotation-text. For instance, the protein $\mathrm{T} 8$, described in the previous section will be encoded as:

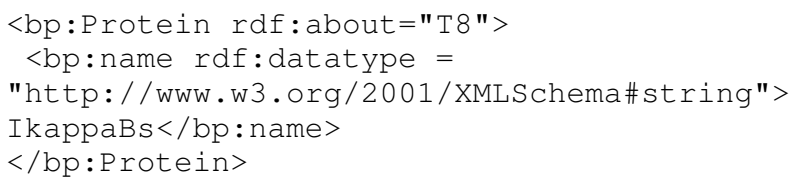

\begin{tabular}{ll} 
Standoff Entity & BioPAX class \\
\hline Cellular_component & prefix.CellularLocationVocabulary \\
Complex & prefix.Complex \\
DNA & prefix.Dna \\
Drug & prefix.PhysicalEntity \\
Entity & prefix.PhysicalEntity \\
Gene_or_gene_product & prefix.PhysicalEntity \\
Gene_product & prefix.PhysicalEntity \\
Gene & prefix.Gene \\
Ion & prefix.PhysicalEntity \\
Protein & prefix.Protein \\
Receptor & prefix.PhysicalEntity \\
RNA & prefix.Rna \\
Simple_molecule & prefix.SmallMolecule \\
Simple_chemical & prefix.SmallMolecule \\
Tag & prefix.PhysicalEntity \\
\hline prefix = org.biopax.paxtools.model.level3
\end{tabular}

Table 3: Mapping of Annotation-type to BioPAX term.

Step 3: Create bp:Interactions Each event is mapped to the corresponding bp: Interaction class in BioPAX. $\mathrm{bp}$ : Interaction is a superclass used to describe reactions and the relationship between the bp:Physicalentity elements. Depending on the type of the event, an appropriate sub class of the bp: Interaction is chosen. The mapping is described in Table 4. The created bp: Interaction is assigned a unique id which is the same as the annotation-ID of the event in the standoff. Additionally, all interaction which have the bp:ConversionDirection attribute, are set to bp:LEFT_TO_RIGHT. 


\begin{tabular}{ll} 
Standoff Event & BioPAX Class \\
\hline Conversion & prefix.Conversion \\
Acetylation & prefix.BiochemicalReaction \\
Deacetylation & prefix.BiochemicalReaction \\
Methylation & prefix.BiochemicalReaction \\
Demethylation & prefix.BiochemicalReaction \\
Phosphorylation & prefix.BiochemicalReaction \\
Dephosphorylation & prefix.BiochemicalReaction \\
Ubiquitination & prefix.BiochemicalReaction \\
Deubiquitination & prefix.BiochemicalReaction \\
Gene_expression & prefix.TemplateReaction \\
Transcription & prefix.TemplateReaction \\
Translation & prefix.TemplateReaction \\
Catalysis & prefix.Catalysis \\
Degradation & prefix.Degradation \\
Catabolism & prefix.Degradation \\
Protein_catabolism & prefix.Degradation \\
Association & prefix.ComplexAssembly \\
Binding & prefix.ComplexAssembly \\
Dissociation & prefix.ComplexAssembly \\
Regulation & prefix.Control \\
Positive_regulation & prefix.Catalysis \\
Activation & prefix.Control \\
Negative_regulation & prefix.Control \\
Inactivation & prefix.Control \\
Localization & prefix.Transport \\
Transport & prefix.Transport \\
\hline prefix = org.biopax.paxtools.model.level3
\end{tabular}

Table 4: Mapping of annotation-type to BioPAX interaction class.

Step 4: Add participants Events relevant for this paper fall into 3 categories 1) bp: TemplateReaction (for transcription ,translation and Gene_expression events), 2) $\mathrm{bp}$ :Conversion (for conversion events including phosphorylation, dephosphorylation etc., transport events, binding events and dissociation events) and 3) bp:Control (for regulation, positive regulation, activation, negative regulation and inactivation events).

Gene Expression, Transcription and Translation events are modeled as a bp:TemplateReaction. If the Theme of a transcription event is of type RNA, then it is mapped to the bp:product property of the bp:TemplateReaction. If the Theme is a DNA, then it is added as bp:template property. Similarly, if the Theme of a Gene expression event (Translation or Transcription) is of type Protein, then the corresponding bp:Physicalentity is set as the bp:product of the bp:TemplateReaction. If the Theme of a Translation event is an RNA, then it is set as the bp: template property.

Conversion events are easily mapped to BioPAX elements. Conversion events are all modeled as bp:BiochemicalReaction. The bp:Physicalentity corresponding to Theme is set to the bp:left of the bp:BiochemicalReaction. Site information is encoded into the suitable $\mathrm{bp}$ : sequencesite property.

For instance, in the case of a Phosphorylation event, the reaction corresponds to Theme becoming phosphorylated. For this a new bp:Physicalentity is created which has the same properties as Theme, except that it has an additional bp:ModificationFeature, which corresponds to the phosphorylated residue. This new entity is then set to bp:right of the bp:BiochemicalReaction. If these reactions have the Cause entity, then, a new $\mathrm{bp}$ :Control interaction is created with the Cause entity as the bp:controller and the created bp:BiochemicalReaction as the bp: controlled.

Similarly, Binding, Dissociation and Degradation events map from their definitions onto the BioPAX setting.

Localization and transport events with the TOLOC and FromLOC roles are handled differently. The ToLOC and FromLoc entities are added as compartments in the BioPAX model. We then model a bp:Transport reaction with the Theme entity transported from the FromLoc compartment to the ToLoc compartment. Localization events with AtLOC role are not explicitly modeled as reaction. Only the compartment of the corresponding Theme's bp: Physicalentity in the BioPAX model is appropriately set. Additionally, the annotation-ID of the event is appended as a comment to the corresponding element in BioPAX.

Control events are more complex since they can involve another event as a Theme or Cause. Positive/Negative Regulation, Activation and Inactivation events where Theme is mapped to a bp:Physicalentity are modeled as a bp:BiochemicalReaction. Here the entity is converted from an active/inactive form to an inactive/active form. Next, a corresponding bp:Control interaction is created (see Table 4). If the Cause is also an entity then it is added as the bp:controller to the bp:Control interaction. However, in case Cause is an event, then the right side entity (or product) of the Interaction encoded by the Cause event is derived 
and added as the bp:controller. The previously created bp:BiochemicalReaction is then added as the bp:controlled element for the bp:Control interaction. The bp:controltype property is set to $\mathrm{bp}$ :ACTIVATION and $\mathrm{bp}$ : INHIBITION for the Positive Regulation/Activation and Negative Regulation/Inactivation events respectively.

Regulation, Positive Regulation and Negative Regulation can also have events in the Theme role. In this case, the Interaction corresponding to the Theme is searched, and added as the bp:controlled element of a new $\mathrm{bp}$ :Control interaction. Should there be a Cause entity or event then it is handled as described previously.

\section{Step 5: Optional Postprocessing Operations} The software for BioPAX supports post processing similar to the SBML converter: 1) Unused entities can be removed, 2) interactions completed and 3) interactions without reactants and products removed. Additionally, we can assign a unique identifier to BioPAX entities by querying external databases like UniProt, this information is encoded into the bp:Xref class using either bp:RelationshipXref or bp:UnificationXref.

\subsection{Discussion}

The conversion from standoff to BioPAX is relatively straightforward. The finer grained options to represent different types of information makes it more naturally suited to translate annotations from standoff format. Nevertheless, issues highlighted in the SBML conversion exist in the BioPAX conversion too. For example, certain events such as Localization events with an AtLoc role do not end up as bp: Interaction etc.

\subsection{Implementation}

The algorithm is implemented in python. It uses the Java Paxtools 4.2.1 toolkit (Demir et al., 2013) to encode and manipulate models into the BioPAX format. JPype is used as the bridge to connect python to the Paxtools library. The other components of the implementation (such as the standoffparser) are the same as used in the SBML implementation.

\section{Results and Evaluation}

For initial evaluation of our software we used the mTOR pathway event corpus also used in a related study on converting pathway models to standoff format (Ohta et al., 2011). The corpus consists of 60 PubMed abstracts and the same number of files of hand-annotated standoff files. The 60 abstracts contain 11960 words. The hand-annotated data contains 1284 events, 1483 Protein, 1 Entity, 201 Complexes (which gives a total of 2970 text bound annotation triggers). In total the annotations contain 1228 Theme roles, 19 Product roles, 205 Causes, 139 Site, 8 atLoc, 4 fromLoc, 16 toLoc and 51 participant. The conversion run on the hand-annotated data correctly translates entities and events to SBML and BioPAX according to the mapping described in the previous sections.

In order to check our software with state-of-theart event extraction systems we applied an unaltered, freshly downloaded Turku Event Extraction System/TEES Version 2.1 (Björne et al., 2013) to the 60 PubMed abstracts. The resulting TEES/60 corpus contains 1472 text bound triggers (in a1) and 783 text bound triggers (in a2). TEES extracted 1473 Proteins which were all successfully translated to SBML and BioPAX. 20 entities were detected, 3 of which were translated into compartments (based on their usage in Localization), 10 were used as site and translated into site comments. In total 1126 events were detected by TEES of which the majority was translated. The exception were 30 localization events of which 1 was a localization with an At Loc role (translated into a compartment). 29 Localization events were only annotated with a theme and therefore were ignored. 270 regulation events have an event based theme. Only 99 of those are also cause annotated and handled as sbml:reaction. The remaining 171 disappear since the extracted information from TEES is not enough to establish links in the models (both BioPAX and SBML).

Importantly, the failure to translate some of these events into SBML/BioPAX is caused by incomplete information provided by the NLP event extraction system. For instance, Localization events which only have a Theme role do not provide enough information to be added to the model. Obviously this is one of the areas where handannotated data provides better conversion results. Nevertheless, these kind of results are encourag- 
ing because the translation into biological knowledge allows for further processing and cleaning of automatically extracted data and potentially may lead to better extraction systems by providing additional learning signals.

Working with Natural language is never easy. Natural language is full of underspecification, ambiguities and context-dependencies. Standoff formats represent a compromise between exact specifications such as SBML and BioPAX that come with their own design approach and assumptions. Trying to map from one world into the other we noticed a few problems

\section{Coarse type granularity of biological players:}

Coarse granularities such as "Gene or gene product", which encompass genes, RNA and protein, make it difficult to assign a type for the entity. This is important for reactions such as Gene expression, where the decision whether something is a sbml:product or sbml:modifier depends on exact distinctions.

Underspecification of event types: The event type Regulation refers to any process (Cause) that modulates any attribute of another process (Theme). In the pathway representation context, it is more natural that the process that gets modulated be an event (which is modeled as a sbml:reaction in SBML and bp:Interaction in BioPAX). It is not clear how to correctly represent the scenario when the process that gets modulated is an entity (modeled as sbml:species in SBML and bp: PhysicalEntity in BioPAX). However, the event specification allows Theme (that which is regulated) to be either an entity or an event.

Underspecification of roles: Event extraction systems try to extract as much as possible but often are not able to extract all necessary information. For example, the following says there is a Positive_regulation on Theme $\mathrm{T} 23$, but no information is available on the process that is regulating it (no Cause).

E13 Positive_regulation:T36

Theme: T23

In such cases the converter is unable to extract SBML and BioPAX information.

\section{Conclusion}

In this paper we proposed and discussed a scheme to convert NLP event representations to standard biomedical pathway data formats (SBML and BioPAX). This is important for several reasons. The system allows curators to integrate event extraction data into their normal work flow. For instance, the extracted information can give curators a base template, which can be further edited in their favorite drawing tool. The integration into graphical annotation tools could provide the basis to later capture the curator's changes. These changes could in turn be used to generate new human annotations and to improve current event extraction systems. Together with other tools that support the conversion of SBML models into NLP standoff representations (Ohta et al., 2011), our system bridges the gap between biological modeling and automatic event extraction and opens the way to a more tight interaction between the two fields.

Tight integration of NLP and biomedical research is a recent trend (Huang and $\mathrm{Lu}, 2015$ ) with a number of groups moving in this direction (Wei et al., 2013; Cejuela et al., 2014; Miwa et al., 2013, for example). For pathway curation, it is important that the results of event extraction technologies become part of curation applications/workflows. To achieve this we will have to overcome problems inherent in the design of formats such as SBML/BioPAX and/or standoff formats. For instance, SBML wa s primarily developed as process-based transition notation that cannot faithfully capture all known biochemistry. Popular software like CellDesigner add a layer of custom XML annotations to resolve this. For our tools to be used in CellDesigner we have to add such information in the conversion process. Another layer of information can be provided by automatic annotation using UniProt. For the future it will be important to integrate other databases and external references.

Lastly, we plan to perform a more thorough evaluation of the conversion by reconstructing a complete known pathway (e.g. the mTOR pathway, for which high quality maps are already available). We are also performing a large scale evaluation of the software on the EVEX event database - a text mining resource of PubMed abstracts and full texts (Van Landeghem et al., 2011) 


\section{References}

[Ananiadou et al.2010] Sophia Ananiadou, Sampo Pyysalo, Jun'ichi Tsujii, and Douglas B Kell. 2010. Event extraction for systems biology by text mining the literature. Trends in biotechnology, 28(7):381-90, July.

[Björne et al.2013] Jari Björne, Suwisa Kaewphan, and Tapio Salakoski. 2013. Uturku: Drug named entity detection and drug-drug interaction extraction using svm classification and domain knowledge. In Proceedings of the 7th International Workshop on Semantic Evaluation (SemEval 2013).

[Cejuela et al.2014] Juan Miguel Cejuela, Peter McQuilton, Laura Ponting, Steven J Marygold, Raymund Stefancsik, Gillian H Millburn, Burkhard Rost, et al. 2014. tagtog: interactive and textmining-assisted annotation of gene mentions in plos full-text articles. Database, 2014:bau033.

[Demir et al.2010] Emek Demir, Michael P Cary, Suzanne Paley, Ken Fukuda, Christian Lemer, Imre Vastrik, Guanming Wu, Peter D'Eustachio, Carl Schaefer, Joanne Luciano, et al. 2010. The biopax community standard for pathway data sharing. $\mathrm{Na}$ ture biotechnology, 28(9):935-942.

[Demir et al.2013] Emek Demir, Özgün Babur, Igor Rodchenkov, Bülent Arman Aksoy, Ken I Fukuda, Benjamin Gross, Onur Selçuk Sümer, Gary D Bader, and Chris Sander. 2013. Using biological pathway data with paxtools. PLoS computational biology, 9(9):e1003194.

[Funahashi et al.2008] Akira Funahashi, Yukiko Matsuoka, Akiya Jouraku, Mineo Morohashi, Norihiro Kikuchi, and Hiroaki Kitano. 2008. Celldesigner 3.5: a versatile modeling tool for biochemical networks. Proceedings of the IEEE, 96(8):1254-1265.

[Huang and Lu2015] Chung-Chi Huang and Zhiyong Lu. 2015. Community challenges in biomedical text mining over 10 years: success, failure and the future. Briefings in Bioinformatics, page bbv024.

[Hucka et al.2003] Michael Hucka, Andrew Finney, Herbert M Sauro, Hamid Bolouri, John C Doyle, Hiroaki Kitano, Adam P Arkin, Benjamin J Bornstein, Dennis Bray, Athel Cornish-Bowden, et al. 2003. The systems biology markup language (sbml): a medium for representation and exchange of biochemical network models. Bioinformatics, 19(4):524-531.

[Junker et al.2006] Björn H Junker, Christian Klukas, and Falk Schreiber. 2006. Vanted: a system for advanced data analysis and visualization in the context of biological networks. BMC bioinformatics, 7(1):109.

[Kim et al.2011] Jin-Dong Kim, Sampo Pyysalo, Tomoko Ohta, Robert Bossy, Ngan Nguyen, and Jun'ichi Tsujii. 2011. Overview of bionlp shared task 2011. In Proceedings of the BioNLP Shared
Task 2011 Workshop, pages 1-6. Association for Computational Linguistics.

[Le Novere et al.2009] Nicolas Le Novere, Michael Hucka, Huaiyu Mi, Stuart Moodie, Falk Schreiber, Anatoly Sorokin, Emek Demir, Katja Wegner, Mirit I Aladjem, Sarala M Wimalaratne, et al. 2009. The systems biology graphical notation. Nature biotechnology, 27(8):735-741.

[Miwa et al.2013] Makoto Miwa, Tomoko Ohta, Rafal Rak, Andrew Rowley, Douglas B Kell, Sampo Pyysalo, and Sophia Ananiadou. 2013. A method for integrating and ranking the evidence for biochemical pathways by mining reactions from text. Bioinformatics, 29(13):i44-i52.

[Nédellec et al.2013] Claire Nédellec, Robert Bossy, Jin-Dong Kim, Jung-jae Kim, Tomoko Ohta, Sampo Pyysalo, and Pierre Zweigenbaum. 2013. Overview of bionlp shared task 2013. ACL 2013, page 1 .

[Novere et al.2005] Nicolas Le Novere, Andrew Finney, Michael Hucka, Upinder S Bhalla, Fabien Campagne, Julio Collado-Vides, Edmund $\mathbf{J}$ Crampin, Matt Halstead, Edda Klipp, Pedro Mendes, et al. 2005. Minimum information requested in the annotation of biochemical models (miriam). Nature biotechnology, 23(12):15091515.

[Ohta et al.2011] T. Ohta, S. Pyysalo, and J. Tsujii. 2011. From pathways to biomolecular events: Opportunities and challenges. In Proceedings of the BioNLP 2011 Workshop, pages 105-113, Portland, Oregon, USA, June. Association for Computational Linguistics.

[Ohta et al.2013] T. Ohta, S. Pyysalo, R. Rak, A. Rowley, H.-W. Chun, S. J. Jung, S. P. Choi, and S. Ananiadou. 2013. Overview of the pathway curation (pc) task of bionlp shared task 2013. In Proceedings of the BioNLP Shared Task 2013 Workshop, pages 67-75. Association for Computational Linguistics, August.

[Van Landeghem et al.2011] Sofie Van Landeghem, Filip Ginter, Yves Van de Peer, and Tapio Salakoski. 2011. Evex: a pubmed-scale resource for homology-based generalization of text mining predictions. In Proceedings of BioNLP 2011 Workshop, pages 28-37. Association for Computational Linguistics.

[Wei et al.2013] Chih-Hsuan Wei, Hung-Yu Kao, and Zhiyong Lu. 2013. Pubtator: a web-based text mining tool for assisting biocuration. Nucleic acids research, page gkt441. 